\title{
EVALUATION OF INTRADERMAL TRANXEMIC ACID INJECTION IN TREATMENT OF MELASMA
}

By

\section{Mohammed Fawzy Ewaiss, Abd El-Raouf Mohamed El-Mohsen and Emad Mahmoud El-Rewiny}

Department of Dermatology, Venereology and Andrology, Faculty of Medicine, Al-Azhar University, Cairo, Egypt

Corresponding author: Mohammed Fawzy Ewaiss

Mobile: 01060173109, E-mail: dr.fawzyewsiss@ gmail.com

\begin{abstract}
Background: Melasma is an acquired skin disorder characterized by light to dark brown macules and patches occurring in the sun-exposed areas of the face. The major etiological factors include genetic influences, pregnancy, sex hormones, exposure to ultraviolet rays (UVR) radiation and cosmetics. The treatment of melasma remains a challenge. Numerous treatment options as topical agents, systemic depigmenting therapy, chemical peeling and laser have been included in melasma treatment. No single therapy has proven to be of benefit to all patients, combination of modalities can achieve more control especially in difficult cases.
\end{abstract}

Objective: To evaluate the efficacy of intradermal tranexamic acid injection in treatment of melasma.

Patients and Methods: Sixty Egyptian patients presented with melasma were enrolled into our study and divided into 2 equal groups: group A with intradermal injection of tranexamic acid, and group B were injected with intradermal saline. The patients were recruited from the Dermatology Outpatient Clinic of AlAzhar University Hospitals during the period from August 2019 to October 2019.

Results: All patients were females. The mean age was $41.87 \pm 7.79$ years, and the median of duration of melasma among the patients were 7 years. Gravidity and pregnancy (96.7\%) were the most common precipitating factors while family history was positive in $46.7 \%$. According to the efficacy of the therapy in melasma patients, intradermal injection of tranexamic acid showed that the percentage of change of mMASI score after treatment was $58.123 \%$. Response to therapy in melasma, showed very good response in $18.3 \%$, moderate response in $13.3 \% \%$, good response in $16.7 \% \%$, no response in $51.7 \%$. As regard the side effects, tranexamic acid side effects were mild burning and transient edema at the site of injection in whole 60 patients. Five patients showed post inflammatory hyperpigmentation (8.3\%).

Conclusion: Intradermal tranexamic acid is safe, well tolerated and effective therapy in the treatment of melasma. Tranexamic acid solution is available therapy, not expensive and easy to be prepared and applied. Tranexamic acid is preferred in dark skin phototypes than as it is more safe and less liable to cause PIH. Tranexamic acid prevents the rapid relapse of melasma after stoppage of treatment.

Keywords: Tranxemic Acid; Melasma; mMASI.

\section{INTRODUCTION}

$\begin{array}{crr}\text { Melasma is a common } & \text { acquired } \\ \text { condition } & \text { characterized } & \text { by }\end{array}$

hyperpigmented macules or patches most often distributed on the centrofacial (involving the forehead, nose, upper lip, and chin), malar area while other areas could be affected as mandibular area (along the jaw line) (Bala et al., 2018). 
Although melasma may affect both sexes and all races, men account only $10 \%$ of cases, and it is more common among women in childbearing age (30-55 years old). It is also common in Asian who lives in tropical areas. Family history is presented in $50-70 \%$ of cases. The centrofacial pattern is the commonest pattern of melasma in females. However, in men, the malar pattern is the commonest one. These wide ranges are secondary to varieties in predominance among darker skin types, distinctive ethnic heritages, and different levels of UV exposure inside different geographic areas. As such, the genuine predominance across the whole populace is unknown. The age of onset is additionally obscure, with normal age extending between 20 and 30 years (Handel et al., 2014).

Various underlying risk factors have been described including sun exposure, hormonal changes, pregnancy, genetic predisposition and oral contraceptive pills (Sarkar et al., 2018).

Despite being not fully understood making its management still challenging, the pathogenesis of melasma could be due to melanogenesis dysfunction either increased exposure to melanogenic factors or increased sensitivity to previous factor resulting in upregulated melanogenesis and therefore melanosome transfer to keratinocytes through tyrosinase enzyme which is responsible for regulation of melanin production (Zhang et al., 2018).

Managing melasma started first by prevention using sun screens. Therapeutic approaches of Melasma utilizing chemical peal and topical drug as hydroquinone, azelaic acid, retinoids, corticosteroids and arbutin used either alone or in combinations have been commonly employed as the first line of treatment (Sarkar et al., 2017).

Hydroquinone (HQ), also known as dihydroxybenzene, is a hydroxyphenolic compound that is structurally similar to precursors of melanin. It inhibits the conversion of DOPA to melanin by inhibition of the enzyme, tyrosinase. HQ affects not only the formation, melanization, and degradation of melanosomes, but it also affects the membranous structures of melanocytes and eventually causes necrosis of whole melanocytes (Shankar et al., 2014).

On the other hand, approaches based on degree of pigmentation and type epidermal, dermal or mixed and using laser is debatable. Tranexamic acid (TXA) may be one of promising treatment for melasma, allowing more effective treatment with fewer side effects and lower recurrence rate (Khurana et al., 2019).

Tranexamic acid is a synthetic derivative of the amino acid lysine was first described in 1979 when Nijo Sadako discovered its effect incidentally while treating a patient for chronic urticaria (Perper et al., 2017). It has antifibrinolytic effects. FDA approved as a hemostatic agent for heavy bleeding and for patients with hemophilia.

Tranexamic acid acts on melasma by inhibiting UV-induced plasmin activity in keratinocytes. So, blocking the binding of plasminogen to keratinocytes, thereby decreasing the free arachidonic acid which is the cornerstone of prostaglandins production for up-grading of tyrosinase activity (Sheu, 2018). 


\section{EVALUATION OF INTRADERMAL TRANXEMIC ACID INJECTION IN... ${ }^{657}$}

Furthermore, Sharma et al. (2017) founded that TXA decreases the vascular element of melasma by inhibition of freely diffusible forms of vascular endothelial growth factor (VEGF), thus decreasing the response for angiogenesis and minimizing erythema.

The present workd aimed to evaluate the efficacy of intradermal tranexamic acid injection in treatment of melasma.

\section{PATIENTS AND METHODS}

Sixty Egyptian patients presented with melasma were enrolled into our study and divided into 2 equal groups: group A with intradermal injection of tranexamic acid, and group $\mathrm{B}$ were injected with intradermal saline. The patients were recruited from the Dermatology Outpatient Clinic of Al-Azhar University Hospitals during the period from August 2019 to October 2019. Informed written consents obtained from all patients. The approval from the Research Ethics Committee of the Faculty of Medicine, Al-Azhar University was also obtained.

Patients were included in the study according to the following criteria: Female patients, age $\geq 18$ with melasma. Exclusion criteria were: pregnancy, lactation, women on hormonal contraceptive, patients on any concurrent therapy for melasma (none of the patients should have received any treatment for melasma 1 month before study entry), patients with hypersensitivity to tranexamic acid, endocrinology diseases, hormone replacement therapy, and family or personal history of one of the following: autoimmune disorders, coagulation disorders, chronic venous insufficiency, heart diseases, retinopathies and kidney disorders.

Each patient was subjected to full history taking and general examination.

\section{Dermatological examination:}

Clinical assessment was performed for all patients:

- Lesion distribution: The cases were differentiated into: centrofacial, malar, and there were no cases of mandibular pattern of melasma.

- Fitzpatrick skin phototype detection: depending on the Fitzpatrick scale.

- Wood's lamp examination: was done for all patients to determine the type of melasma (epidermal, dermal and mixed). The used device was Waldmann DHL404. Patients advised to cover their eyes before examination to avoid retinal injury.

Melasma types were detected into the following: Epidermal type: where pigmentation was intensified under Wood's light examination, Dermal type: where pigmentation weren't intensified under Wood's light examination and Mixed type: were pigmentation became more apparent in some areas while in others there was no change under the Wood's light.

Frontal, right, and left views photographs were taken for every patient at (baseline, the end of treatment and 3 months after the last session). These clinical images were taken using standardized high-resolution digital photographs using identical camera settings and position. 


\section{Therapeutic regimen:}

- Cleansing the face with alcohol.

- The patients received a topical anesthetic agent for $30 \mathrm{~min}$ (Emla cream; lidocaine $25 \mathrm{mg}$, prilocaine 25 mg, Astra Xeneca, Mississauga, Canada).

- Study Group A (tranexamic acid): were intradermally microinjected with 1-2 $\mathrm{ml}$ of tranexamic acid solution in normal saline $(4 \mathrm{mg} / \mathrm{ml})$ into the melasma lesion at $1 \mathrm{~cm}$ interval by using sterile insulin syringe every week.

- Control (saline) group B: were intradermally injected with $1 \mathrm{~cm}$ intervals by an insulin syringe with a 30-gauge needle every week.

- Clinical changes or adverse reactions were recorded.

\section{Preparation of the used agents:}

$1 \mathrm{~mL}$ TXA (KAPRON ${ }^{\circledR}$ ampoules, tranexamic acid $100 \mathrm{mg} / \mathrm{ml}$, Amoun, Cairo, Egypt). Tranexamic acid is available as a $5 \mathrm{~mL}$ vial containing 500 mg. An insulin syringe was used, with a volume of $1 \mathrm{~mL}$, containing $0.04 \mathrm{~mL}$ (4 units of insulin) of TXA and the remainder being normal saline; thus each insulin syringe contains $4 \mathrm{mg}$ TA. In order to prepare the drug at a concentration of
$10 \mathrm{mg}, 0.1 \mathrm{~mL}$ (10 units of insulin) of the TXA was required to reach a volume of 1 $\mathrm{mL}$ with normal saline. Freshly prepared formulation was delivered to patients every session.

\section{Statistical Analysis:}

Data were collected, revised, coded and entered to the Statistical Package for Social Science (IBM SPSS) version 23. The quantitative data were presented as mean, standard deviations and ranges when parametric and median, interquartile range (IQR) when data found non-parametric. Also qualitative variables were presented as number and percentages. The Comparison between groups with qualitative data were done by using Chi-square test. The comparison between two groups with quantitative data and parametric distribution were done by using Independent t-test; while data with non-parametric distribution were done by using Mann-Whitney test. The comparison between more than two independent groups with quantitative data and parametric distribution was done by using One Way Analysis of Variance (ANOVA). While data with nonparametric distribution were done by using Kruskall wallis test. The confidence interval was set to $95 \%$ and the margin of error accepted was set to $5 \%$. So, $\mathrm{P}$ values were considered significant at $\leq 0.05$. 
EVALUATION OF INTRADERMAL TRANXEMIC ACID INJECTION IN... 659

\section{RESULTS}

Regarding to the present study the ages of patients ranged from 29 - 56years old, the mean age was $42.13 \pm 6.8$ years, and the duration of melasma among the patients ranged from 3 - 12years with a median of 5.0 years.

Regarding the different precipitating factors, Gravidity and Pregnancy were taking the upper hand as precipitating factors of melasma in 58 patients $(96.7 \%)$, followed by sun exposure as an important factor in 58 patients $(96.7 \%)$ also family history was presented in 28 patients (46.7\%).

According to Fitzpatrick skin phototype, there were twenty six patients (43.4\%) with skin phototype III, twenty patients $(33.3 \%)$ with skin phototype IV, and fourten patients (23.3\%) with skin phototype V. Regarding the pattern of melasma, there were fifty two patient $(86.7 \%)$ with Centro facial pattern of melasma, and eight patients $(13.3 \%)$ with malar pattern. Considering the Wood's light examination, there were fourty four patients $(73.3 \%)$ had epidermal melasma, while sixteen patients had mixed melasma type $(26.7 \%)$ (Table 1).

Regarding the modified MASI (mMASI) score before and after treatment with intradermal injection of tranexamic acid on the group $\mathrm{A}$, the
mMASI score before treatment ranged from $0.60-8.50$ with a median of 3.60 and the mMASI score after treatment ranged from $0.30-8.50$ with a median of 2.05. There was a statistically significant decrease in mMASI score after treatment with intradermal tranexamic acid ( $p$ value $\left.<0.001^{*}\right)$. Considering the relapse of melasma after treatment on the right side, the mMASI score after 3 months from the last session ranged from 0.3 to 8.1 with a median of 1.38 .

As regard the response of treatment in every patient: No response: occurred in thirty one patients $(51.7 \%)$. Moderate response: occurred in eight patients $(13.3 \%)$. Good response: occurred in ten patients $(16.7 \%)$. Very good response: occurred in eleven patients (18.3\%). As regard the Satisfaction in every patient: Poor Satisfaction: occurred in thirty two patients (53.3\%). Good Satisfaction: occurred in sixteen patienst (13.3\%). Very good Satisfaction: occurred in ten patients $(26.7 \%)$. Excellent Satisfaction: occurred in two patients $(3.3 \%)$.

The whole sixty patients $(100 \%)$, suffered from burning sensation and mild edema during the intradermal injection, five patients showed post inflammatory hyperpigmentation $(8.3 \%)$. 
Table (1): Descriptive data regarding age, duration of melasma, mMASI before and after, percentage of improvement, response, satisfaction to treatment, risk factor, Fitz skin type, Pattern of melasma, Wood's light and side effect among the studied cases.

\begin{tabular}{|c|c|c|}
\hline \multirow{3}{*}{ Age (Years) } & & No. $=60$ \\
\hline & Mean \pm SD & $41.87 \pm 7.79$ \\
\hline & Range & $29-56$ \\
\hline \multirow{2}{*}{ Duration of melasma (Years) } & Median(IQR) & $5.00(3-12)$ \\
\hline & Range & $1.0-28.00$ \\
\hline \multirow{2}{*}{ mMASI before } & Median(IQR) & $3.60(1.85-4.6)$ \\
\hline & Range & $0.60-8.50$ \\
\hline \multirow{2}{*}{ mMASI after } & Median(IQR) & $2.05(0.98-3.75)$ \\
\hline & Range & $0.30-8.50$ \\
\hline \multirow{2}{*}{ \%of improvement } & Median(IQR) & $0(0-56.5)$ \\
\hline & Range & $0-91.60$ \\
\hline \multirow{4}{*}{ Category } & No & $31(51.7 \%)$ \\
\hline & Good & $10(16.7 \%)$ \\
\hline & Moderate & $8(13.3 \%)$ \\
\hline & Very good & $11(18.3 \%)$ \\
\hline \multirow{4}{*}{ Satisfaction } & Poor & $32(53.3 \%)$ \\
\hline & Good & $16(26.7 \%)$ \\
\hline & Very good & $10(16.7 \%)$ \\
\hline & Excellent & $2(3.3 \%)$ \\
\hline \multicolumn{2}{|l|}{ Gravidity } & $58(96.7 \%)$ \\
\hline \multicolumn{2}{|l|}{ Pregnancy } & $46(76.7 \%)$ \\
\hline \multicolumn{2}{|c|}{ OCP/Hormones } & $12(20.0 \%)$ \\
\hline \multicolumn{2}{|l|}{ Thyroid } & $0(0.0 \%)$ \\
\hline \multicolumn{2}{|c|}{ Family history } & $28(46.7 \%)$ \\
\hline \multicolumn{2}{|c|}{ Sun exposure } & $58(96.7 \%)$ \\
\hline \multicolumn{2}{|c|}{ Emotional stress } & $30(50.0 \%)$ \\
\hline \multicolumn{2}{|c|}{ Family history } & $28(46.7 \%)$ \\
\hline \begin{tabular}{cc}
\multicolumn{2}{c}{ Fitz skin typ } \\
$\bullet$ & II \\
$\bullet$ & IV \\
$\bullet$ & V
\end{tabular} & & $\begin{array}{l}26(43.3 \%) \\
20(33.3 \%) \\
14(23.3 \%)\end{array}$ \\
\hline \multicolumn{2}{|c|}{$\begin{array}{cc}- & \text { Malar } \\
- & \text { Centro facial }\end{array}$} & $\begin{array}{l}8(13.3 \%) \\
52(86.7)\end{array}$ \\
\hline \multicolumn{2}{|c|}{$\begin{array}{cc}\text { Wood's light: } \\
\text { - } & \text { Epidermal } \\
\text { Mixed } & \text { Mixed }\end{array}$} & $\begin{array}{l}44(73.3 \%) \\
16(26.7 \%)\end{array}$ \\
\hline \multicolumn{2}{|c|}{ 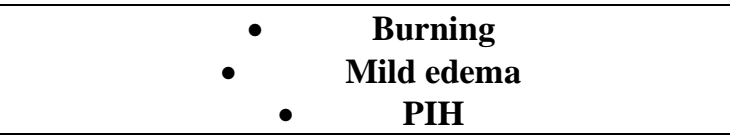 } & $\begin{array}{c}60(100 \%) \\
60(100 \%) \\
5(8.3 \%)\end{array}$ \\
\hline
\end{tabular}




\section{EVALUATION OF INTRADERMAL TRANXEMIC ACID INJECTION IN... ${ }^{661}$}

According to table (2), there was no significant relation between age and response to treatment. Also, no significant relation between response to treatment and precipitating factor, mMASI before and PIH.
There was significant relation between response to treatment and duration of melasma and between response to treatment and mMASI after, $\%$ of improvement and satisfaction.

Table (2): Relation between response to treatment regarding age, duration of melasma, mMASI before and after, percentage of improvement, satisfaction to treatment, PIH, risk factor among the studied cases

\begin{tabular}{|c|c|c|c|c|c|c|}
\hline \multirow{2}{*}{\multicolumn{2}{|c|}{ Parameters $\quad$ Grades }} & No & Good & Moderate & Very good & \multirow{2}{*}{ P-value } \\
\hline & & No. $=31$ & No. $=10$ & No. $=8$ & No. $=11$ & \\
\hline \multirow{2}{*}{ Age (Years) } & Mean \pm SD & $41.81 \pm 7.73$ & $39.30 \pm 5.54$ & $48.25 \pm 8.89$ & $39.73 \pm 7.18$ & \multirow{2}{*}{$0.059 \bullet$} \\
\hline & Range & $29-56$ & $32-48$ & $30-56$ & $29-54$ & \\
\hline \multirow{2}{*}{$\begin{array}{c}\text { Duration of } \\
\text { melasma } \\
\text { (years) }\end{array}$} & $\begin{array}{l}\text { Median } \\
\text { (IQR) }\end{array}$ & $5.00(3-12)$ & $5.00(3-6)$ & $\begin{array}{c}18.50(13- \\
21.5) \\
\end{array}$ & $5.00(3-8)$ & \multirow[t]{2}{*}{$0.026+$} \\
\hline & Range & $1.00-28.00$ & $1.00-20.00$ & $3.00-28.00$ & $1.00-12.00$ & \\
\hline \multirow{2}{*}{ mMASI before } & $\begin{array}{l}\text { Median } \\
\text { (IQR) }\end{array}$ & $\begin{array}{c}3.60(1.85- \\
4.6)\end{array}$ & $\begin{array}{c}3.38(1.85- \\
4.6)\end{array}$ & $\begin{array}{l}4.88(2.7- \\
6.23)\end{array}$ & $2.70(1.8-3.6)$ & \multirow[t]{2}{*}{0.244} \\
\hline & Range & $0.60-8.50$ & $0.60-5.50$ & $1.20-8.50$ & $1.20-4.50$ & \\
\hline \multirow[t]{2}{*}{ mMASI after } & $\begin{array}{c}\text { Median } \\
\text { (IQR) }\end{array}$ & $\begin{array}{c}3.60(1.85- \\
4.6)\end{array}$ & $1.23(0.65-2)$ & $\begin{array}{c}3.40(1.8- \\
4.05)\end{array}$ & $0.35(0.3-0.6)$ & \multirow[t]{2}{*}{0.000} \\
\hline & Range & $0.60-8.50$ & $0.30-2.15$ & $0.90-6.35$ & $0.30-1.05$ & \\
\hline \multirow{2}{*}{$\begin{array}{c}\text { \%of } \\
\text { improvement }\end{array}$} & $\begin{array}{c}\text { Median } \\
\text { (IQR) }\end{array}$ & $0.00(0-0)$ & $\begin{array}{l}55.75(50.5- \\
63.6)\end{array}$ & $\begin{array}{c}33.17(26.07- \\
34.67)\end{array}$ & $\begin{array}{c}83.30(75- \\
88.8)\end{array}$ & \multirow[t]{2}{*}{0.000} \\
\hline & Range & $0.00-0.00$ & $50.00-74.07$ & $25.20-42.20$ & $75.00-91.60$ & \\
\hline \multirow{4}{*}{ Satisfaction } & Poor & $30(96.8 \%)$ & $1(10.0 \%)$ & $0(0.0 \%)$ & $1(9.1 \%)$ & \multirow{4}{*}{0.000} \\
\hline & Good & $1(3.2 \%)$ & $3(30.0 \%)$ & $7(87.5 \%)$ & $5(45.5 \%)$ & \\
\hline & Very good & $0(0.0 \%)$ & $6(60.0 \%)$ & $1(12.5 \%)$ & $3(27.3 \%)$ & \\
\hline & \begin{tabular}{|l|} 
Excellent \\
\end{tabular} & $0(0.0 \%)$ & $0(0.0 \%)$ & $0(0.0 \%)$ & $2(18.2 \%)$ & \\
\hline \multirow{2}{*}{$\mathrm{PIH}$} & No & $29(93.5 \%)$ & $8(80.0 \%)$ & $8(100.0 \%)$ & $10(90.9 \%)$ & \multirow{2}{*}{0.447} \\
\hline & Yes & $2(6.5 \%)$ & $2(20.0 \%)$ & $0(0.0 \%)$ & $1(9.1 \%)$ & \\
\hline \multicolumn{2}{|c|}{ Gravidity } & $30(96.8 \%)$ & $9(90.0 \%)$ & $8(100.0 \%)$ & $11(100.0 \%)$ & 0.565 \\
\hline \multicolumn{2}{|c|}{ pregnancy } & $24(77.4 \%)$ & $7(70.0 \%)$ & $6(75.0 \%)$ & $9(81.8 \%)$ & 0.933 \\
\hline \multicolumn{2}{|c|}{ OCP/Hormones } & $6(19.4 \%)$ & $0(0.0 \%)$ & $2(25.0 \%)$ & $4(36.4 \%)$ & 0.215 \\
\hline \multicolumn{2}{|c|}{ Thyroid } & $0(0.0 \%)$ & $0(0.0 \%)$ & $0(0.0 \%)$ & $0(0.0 \%)$ & $\mathrm{NA}$ \\
\hline \multicolumn{2}{|c|}{ Family history } & $14(45.2 \%)$ & $6(60.0 \%)$ & $1(12.5 \%)$ & $7(63.6 \%)$ & 0.123 \\
\hline \multicolumn{2}{|c|}{ Sun exposure } & $30(96.8 \%)$ & $9(90.0 \%)$ & $8(100.0 \%)$ & $11(100.0 \%)$ & 0.565 \\
\hline \multicolumn{2}{|c|}{ Emotional stress } & $16(51.6 \%)$ & $4(40.0 \%)$ & $5(62.5 \%)$ & $5(45.5 \%)$ & 0.796 \\
\hline \multicolumn{2}{|c|}{ Family history } & $15(48.4 \%)$ & $4(40.0 \%)$ & $3(37.5 \%)$ & $6(54.5 \%)$ & 0.859 \\
\hline
\end{tabular}

*:Chi-square test; $\bullet:$ One Way ANOVA test; + : Kruskal Wallis test

According to table (3), there was no significant relation between age, duration of melasma, precipitating factor, mMASI before, response to treatment, Tranexamic acid and saline.
There was significant relation between mMASI after, mMASI before, \%of improvement, patient satisfaction, $\mathrm{PIH}$, Tranexamic acid and Saline. 
Table (3): Relation between tranexmamic acid and saline regarding age, duration of melasma, mMASI before and after, percentage of improvement, response to treatment, satistifaction to treatment, PIH, risk factor among the studied cases

\begin{tabular}{|c|c|c|c|c|}
\hline \multirow{2}{*}{\multicolumn{2}{|c|}{$\begin{array}{ll}\text { Parameters } & \text { Groups } \\
\end{array}$}} & \multirow{2}{*}{$\begin{array}{c}\text { Tranexamic acid } \\
\text { No. }=30\end{array}$} & \multirow{2}{*}{$\begin{array}{c}\text { Saline } \\
\text { No. }=30\end{array}$} & \multirow{2}{*}{ P-value } \\
\hline & & & & \\
\hline \multirow{2}{*}{ Age (Years) } & Mean \pm SD & $41.87 \pm 7.86$ & $40.80 \pm 7.55$ & \multirow{2}{*}{0.593} \\
\hline & Range & $29-56$ & $29-56$ & \\
\hline \multirow{2}{*}{$\begin{array}{l}\text { Durtion of melisma } \\
\text { (Years) }\end{array}$} & Median(IQR) & $5(3-12)$ & $4(3-15)$ & \multirow{2}{*}{0.618} \\
\hline & Range & $1-28$ & $1-26$ & \\
\hline \multirow{2}{*}{ mMASI before } & Median(IQR) & $3.6(1.85-4.6)$ & $3.5(1.70-4.62)$ & \multirow{2}{*}{0.318} \\
\hline & Range & $0.6-8.5$ & $0.6-8.5$ & \\
\hline \multirow{2}{*}{ mMASI after } & Median(IQR) & $1.05(0.35-2.15)$ & $3.6(1.85-4.6)$ & \multirow{2}{*}{0.001} \\
\hline & Range & $0.3-6.35$ & $0.6-8.5$ & \\
\hline \multirow{2}{*}{ \%of improvement } & Median(IQR) & $56.5(36-76.66)$ & $0(0-0)$ & \multirow{2}{*}{0.001} \\
\hline & Range & $0-91.6$ & $0-0$ & \\
\hline \multirow{4}{*}{ Category } & No & $1(3.3 \%)$ & $30(100.0 \%)$ & \multirow{4}{*}{0.001} \\
\hline & Good & $10(33.3 \%)$ & $0(0.0 \%)$ & \\
\hline & Moderate & $8(26.7 \%)$ & $0(0.0 \%)$ & \\
\hline & Very good & $11(36.7 \%)$ & $0(0.0 \%)$ & \\
\hline \multirow{4}{*}{ Satisfaction } & Poor & $2(6.7 \%)$ & $30(100.0 \%)$ & \multirow{4}{*}{0.001} \\
\hline & Good & $16(53.3 \%)$ & $0(0.0 \%)$ & \\
\hline & Very good & $10(33.3 \%)$ & $0(0.0 \%)$ & \\
\hline & Excellent & $2(6.7 \%)$ & $0(0.0 \%)$ & \\
\hline \multirow{2}{*}{ PIH } & No & $27(90.0 \%)$ & $28(93.3 \%)$ & \multirow{2}{*}{0.64} \\
\hline & Yes & $3(10.0 \%)$ & $2(6.7 \%)$ & \\
\hline \multicolumn{2}{|l|}{ Gravidity } & $30(100.0 \%)$ & $28(93.3 \%)$ & 0.150 \\
\hline \multicolumn{2}{|l|}{ Pregnancy } & $20(66.7 \%)$ & $26(86.7 \%)$ & 0.067 \\
\hline \multicolumn{2}{|l|}{ OCP/Hormones } & $7(23.3 \%)$ & $5(16.7 \%)$ & 0.518 \\
\hline \multicolumn{2}{|l|}{ Family history } & $17(56.7 \%)$ & $11(36.7 \%)$ & 0.120 \\
\hline \multicolumn{2}{|l|}{ Sun exposure } & $28(93.3 \%)$ & $30(100.0 \%)$ & 0.150 \\
\hline \multicolumn{2}{|l|}{ Emotional stress } & $16(53.3 \%)$ & $14(46.7 \%)$ & 0.605 \\
\hline \multicolumn{2}{|l|}{ Family history } & $12(40.0 \%)$ & $16(53.3 \%)$ & 0.301 \\
\hline
\end{tabular}

$\bullet$ : One Way ANOVA test; $\$$ : Kruskal Wallis test

According to table (4), there was no significant relation between ages, duration of melasma, mMASI before, PIH and patient satisfaction, and precipitating factors except OCP and hormones there was significant relation.
Significant relation was found between mMASI after, \% of improvement, response to treatment, patient satisfaction OCP and hormones. 
EVALUATION OF INTRADERMAL TRANXEMIC ACID INJECTION IN... 663

Table (4): Relation between patients satisfaction regarding age, duration of melasma, mMASI before and after, percentage of improvement, response to treatment, PIH and risk factor among the studied cases

\begin{tabular}{|c|c|c|c|c|c|c|}
\hline \multirow{2}{*}{\multicolumn{2}{|c|}{ Parameters }} & $\begin{array}{c}\text { Poor } \\
\text { Satisfaction }\end{array}$ & Good Satisfaction & $\begin{array}{c}\text { Very good } \\
\text { Satisfaction }\end{array}$ & $\begin{array}{c}\text { Excellent } \\
\text { Satisfaction }\end{array}$ & \multirow[t]{2}{*}{ P-value } \\
\hline & & No. $=32$ & No. $=16$ & No. $=10$ & No. $=2$ & \\
\hline \multirow{2}{*}{ Age (Years) } & Mean \pm SD & $41.78 \pm 7.74$ & $43.00 \pm 8.87$ & $40.90 \pm 7.22$ & $39.00 \pm 5.66$ & \multirow{2}{*}{$0.864 \bullet$} \\
\hline & Range & $29-56$ & $30-56$ & $29-54$ & $35-43$ & \\
\hline \multirow{2}{*}{$\begin{array}{l}\text { Duration of } \\
\text { melasma } \\
\text { (Years) }\end{array}$} & $\begin{array}{l}\text { Median } \\
\text { (IQR) }\end{array}$ & $5.50(3-12)$ & $7.00(5-19)$ & $4.00(1-5)$ & $6.00(3-9)$ & \multirow[t]{2}{*}{$0.103 \%$} \\
\hline & Range & $1.00-28.00$ & $3.00-28.00$ & $1.00-15.00$ & $3.00-9.00$ & \\
\hline \multirow[t]{2}{*}{ Before } & $\begin{array}{l}\text { Median } \\
\text { (IQR) }\end{array}$ & $3.15(1.83-4.55)$ & $3.90(3-4.88)$ & $2.70(1.2-4.05)$ & $3.15(1.8-4.5)$ & \multirow[t]{2}{*}{0.437} \\
\hline & Range & $0.60-8.50$ & $0.60-8.50$ & $1.20-5.50$ & $1.80-4.50$ & \\
\hline \multirow[t]{2}{*}{ After } & $\begin{array}{l}\text { Median } \\
(\mathrm{IQR})\end{array}$ & $3.15(1.8-4.55)$ & $2.05(0.6-3.55)$ & $0.78(0.3-1.2)$ & $0.68(0.3-1.05)$ & \multirow[t]{2}{*}{0.001} \\
\hline & Range & $0.30-8.50$ & $0.30-6.35$ & $0.30-2.15$ & $0.30-1.05$ & \\
\hline \multirow{2}{*}{$\begin{array}{l}\% \text { of } \\
\text { improvement }\end{array}$} & $\begin{array}{l}\text { Median } \\
(\mathrm{IQR})\end{array}$ & $0.00(0-0)$ & $46.10(30.04-78.29)$ & $64.20(55-75)$ & $79.98(76.66-83.3)$ & \multirow[t]{2}{*}{0.001} \\
\hline & Range & $0.00-75.00$ & $0.00-91.60$ & $36.00-88.88$ & $76.66-83.30$ & \\
\hline \multirow{4}{*}{ Category } & No & $30(93.8 \%)$ & $1(6.3 \%)$ & $0(0.0 \%)$ & $0(0.0 \%)$ & \multirow{4}{*}{0.001} \\
\hline & Good & $1(3.1 \%)$ & $3(18.8 \%)$ & $6(60.0 \%)$ & $0(0.0 \%)$ & \\
\hline & Moderate & $0(0.0 \%)$ & $7(43.8 \%)$ & $1(10.0 \%)$ & $0(0.0 \%)$ & \\
\hline & $\begin{array}{l}\text { Very } \\
\text { good }\end{array}$ & $1(3.1 \%)$ & $5(31.3 \%)$ & $3(30.0 \%)$ & $2(100.0 \%)$ & \\
\hline \multirow{2}{*}{$\mathrm{PIH}$} & No & $29(90.6 \%)$ & $14(87.5 \%)$ & $10(100.0 \%)$ & $2(100.0 \%)$ & \multirow{2}{*}{0.682} \\
\hline & Yes & $3(9.4 \%)$ & $2(12.5 \%)$ & $0(0.0 \%)$ & $0(0.0 \%)$ & \\
\hline \multicolumn{2}{|l|}{ Gravidity } & $30(93.8 \%)$ & $16(100.0 \%)$ & $10(100.0 \%)$ & $2(100.0 \%)$ & 0.613 \\
\hline \multicolumn{2}{|l|}{ Pregnancy } & $24(75.0 \%)$ & $14(87.5 \%)$ & $6(60.0 \%)$ & $2(100.0 \%)$ & 0.353 \\
\hline \multicolumn{2}{|c|}{ OCP/Hormones } & $6(18.8 \%)$ & $3(18.8 \%)$ & $1(10.0 \%)$ & $2(100.0 \%)$ & 0.034 \\
\hline \multicolumn{2}{|l|}{ Thyroid } & $0(0.0 \%)$ & $0(0.0 \%)$ & $0(0.0 \%)$ & $0(0.0 \%)$ & NA \\
\hline \multicolumn{2}{|c|}{ Family history } & $14(43.8 \%)$ & $5(31.3 \%)$ & $7(70.0 \%)$ & $2(100.0 \%)$ & 0.106 \\
\hline \multicolumn{2}{|l|}{ Sun exposure } & $31(96.9 \%)$ & $16(100.0 \%)$ & $9(90.0 \%)$ & $2(100.0 \%)$ & 0.572 \\
\hline \multicolumn{2}{|c|}{ Emotional stress } & $17(53.1 \%)$ & $9(56.3 \%)$ & $4(40.0 \%)$ & $0(0.0 \%)$ & 0.428 \\
\hline \multicolumn{2}{|c|}{ Family history } & $15(46.9 \%)$ & $7(43.8 \%)$ & $5(50.0 \%)$ & $1(50.0 \%)$ & 0.991 \\
\hline
\end{tabular}

*:Chi-square test; $\bullet$ : One Way ANOVA test; + : Kruskal Wallis test

There was no significant relation between age, duration of melasma, mMASI before, mMASI after, $\%$ of improvement and PIH and no PIH, PIH, No PIH and precipitating factors except family history (Table 5). 
Table (5): Relation between no PIH and PIH regarding age, duration of melasma, mMASI before and after, percentage of improvement and risk factor among the studied cases

\begin{tabular}{|c|c|c|c|c|}
\hline \multirow{2}{*}{\multicolumn{2}{|c|}{$\begin{array}{ll}\text { Parameters } & \text { Groups } \\
\end{array}$}} & \multirow{2}{*}{$\begin{array}{c}\text { No PIH } \\
\text { No. }=55\end{array}$} & \multirow{2}{*}{$\begin{array}{c}\text { PIH } \\
\text { No. }=5\end{array}$} & \multirow{2}{*}{ P-value } \\
\hline & & & & \\
\hline \multirow{2}{*}{ Age (Years) } & Mean \pm SD & $41.82 \pm 7.76$ & $42.40 \pm 9.10$ & \multirow{2}{*}{$0.875 \bullet$} \\
\hline & Range & $29-56$ & $32-56$ & \\
\hline \multirow{2}{*}{$\begin{array}{l}\text { Duration of } \\
\text { melasma (Years) }\end{array}$} & Median(IQR) & $5.00(3-12)$ & $5.00(5-12)$ & \multirow{2}{*}{$0.808 \%$} \\
\hline & Range & $1.00-28.00$ & $3.00-19.00$ & \\
\hline \multirow{2}{*}{ Before } & Median(IQR) & $3.60(1.85-4.8)$ & $3.60(1.8-4.25)$ & \multirow{2}{*}{0.707} \\
\hline & Range & $0.60-8.50$ & $1.20-4.60$ & \\
\hline \multirow{2}{*}{ After } & Median(IQR) & $2.15(0.9-4.05)$ & $2.00(1.8-2.1)$ & \multirow{2}{*}{0.592} \\
\hline & Range & $0.30-8.50$ & $0.30-3.60$ & \\
\hline \multirow{2}{*}{ \%of improvement } & Median(IQR) & $0.00(0-56.5)$ & $50.50(0-56.5)$ & \multirow{2}{*}{0.604} \\
\hline & Range & $0.00-91.60$ & $0.00-75.00$ & \\
\hline \multicolumn{2}{|l|}{ Gravidity } & $53(96.4 \%)$ & $5(100.0 \%)$ & 0.665 \\
\hline \multicolumn{2}{|l|}{ Pregnancy } & $41(74.5 \%)$ & $5(100.0 \%)$ & 0.198 \\
\hline \multicolumn{2}{|l|}{ OCP/Hormones } & $12(21.8 \%)$ & $0(0.0 \%)$ & 0.243 \\
\hline \multicolumn{2}{|l|}{ Thyroid } & $0(0.0 \%)$ & $0(0.0 \%)$ & NA \\
\hline \multicolumn{2}{|l|}{ Family history } & $28(50.9 \%)$ & $0(0.0 \%)$ & 0.029 \\
\hline \multicolumn{2}{|l|}{ Sun exposure } & $53(96.4 \%)$ & $5(100.0 \%)$ & 0.665 \\
\hline \multicolumn{2}{|l|}{ Emotional stress } & $28(50.9 \%)$ & $2(40.0 \%)$ & 0.64 \\
\hline \multicolumn{2}{|l|}{ Family history } & $25(45.5 \%)$ & $3(60.0 \%)$ & 0.533 \\
\hline
\end{tabular}

*:Chi-square test; $\bullet:$ Independent $\mathrm{t}$-test; + : Mann Whitney test

There was no significant relation between \%improving and precipitating factors (Table 6).

Table (6): Relation between percentage of improvement regarding risk factor among the studied cases

\begin{tabular}{|c|c|c|c|c|}
\hline \multicolumn{2}{|c|}{$\begin{array}{l}\text { \%of improvement } \\
\text { Parameters }\end{array}$} & Median (IQR) & Range & P-value \\
\hline \multirow{2}{*}{ Gravidity } & No & $25.96(0-51.92)$ & $0-51.92$ & \multirow{2}{*}{0.929} \\
\hline & Yes & $0.00(0-56.50)$ & $0-91.60$ & \\
\hline \multirow{2}{*}{ Pregnancy } & No & $13.54(0-55.00)$ & $0-88.80$ & \multirow{2}{*}{0.970} \\
\hline & Yes & $0.00(0-56.50)$ & $0-91.60$ & \\
\hline \multirow{2}{*}{ OCP/Hormones } & No & $0.00(0-55.75)$ & $0-91.60$ & \multirow{2}{*}{0.758} \\
\hline & Yes & $13.54(0-75.83)$ & $0-83.30$ & \\
\hline \multirow{2}{*}{ Family history } & No & $0.00(0-46.35)$ & $0-91.60$ & \multirow{2}{*}{0.367} \\
\hline & Yes & $16.67(0-74.54)$ & $0-88.80$ & \\
\hline \multirow{2}{*}{ Sun exposure } & No & $28.25(0-56.50)$ & $0-56.50$ & \multirow{2}{*}{0.982} \\
\hline & Yes & $0.00(0-56.50)$ & $0-91.60$ & \\
\hline \multirow{2}{*}{ Emotional stress } & No & $12.60(0-56.50)$ & $0-91.60$ & \multirow{2}{*}{0.644} \\
\hline & Yes & $0.00(0-56.50)$ & $0-83.33$ & \\
\hline \multirow{2}{*}{ Family history } & No & $12.60(0-56.50)$ & $0-91.60$ & \multirow{2}{*}{0.949} \\
\hline & Yes & $0.00(0-64.54)$ & $0-88.88$ & \\
\hline
\end{tabular}


EVALUATION OF INTRADERMAL TRANXEMIC ACID INJECTION IN... 665

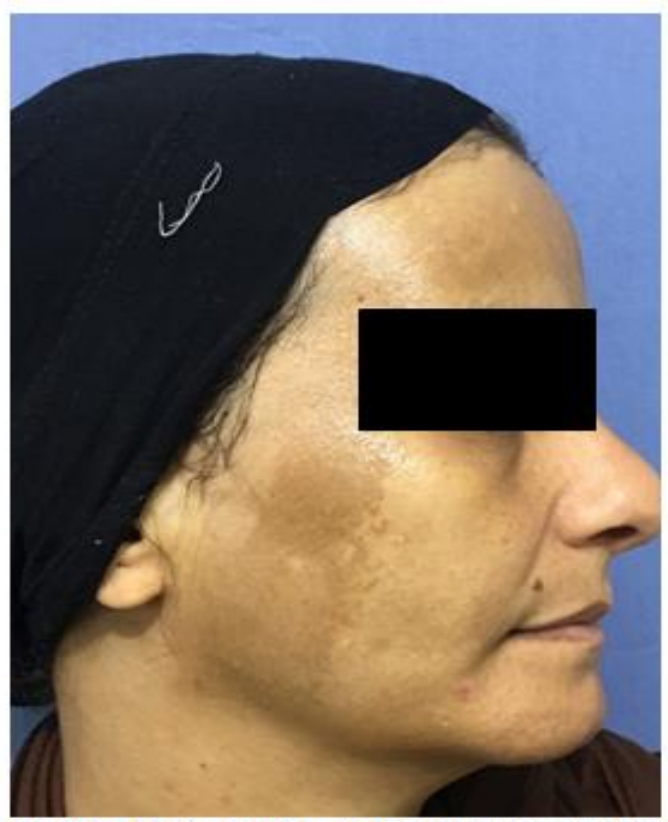

Photo (la): Right side melasma before treatment

Improvement of melasma after 12 weeks tranexamic acid intradual injection. Clinical photographs were taken at week 0

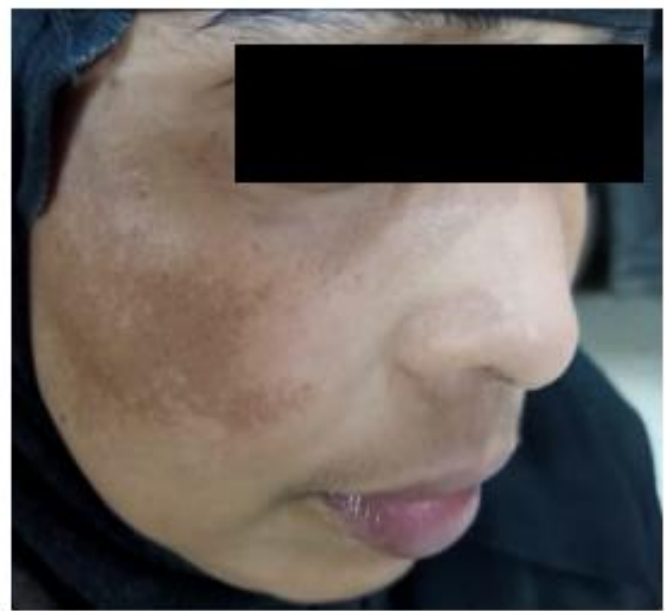

Photo (2a): Right side melasma before treatment

Improvement of melasma after 12 weeks tranexamic acid intradual injection. Clinical photographs were taken at week 0

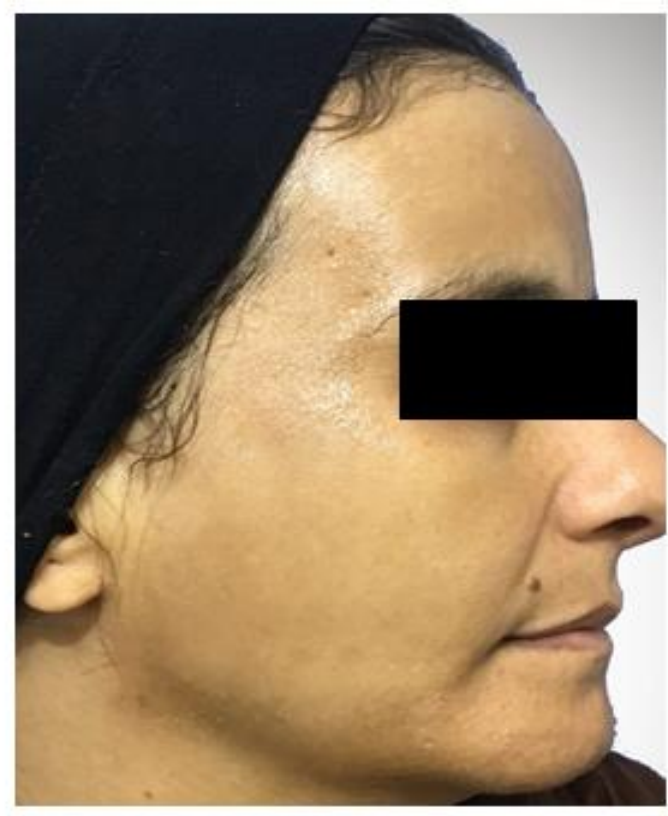

Photo (lb): Right side melasma at the end of the treatment with very good response

and week 12, improvement of pigmentation $(a, b)$ is noticeable with very good response.

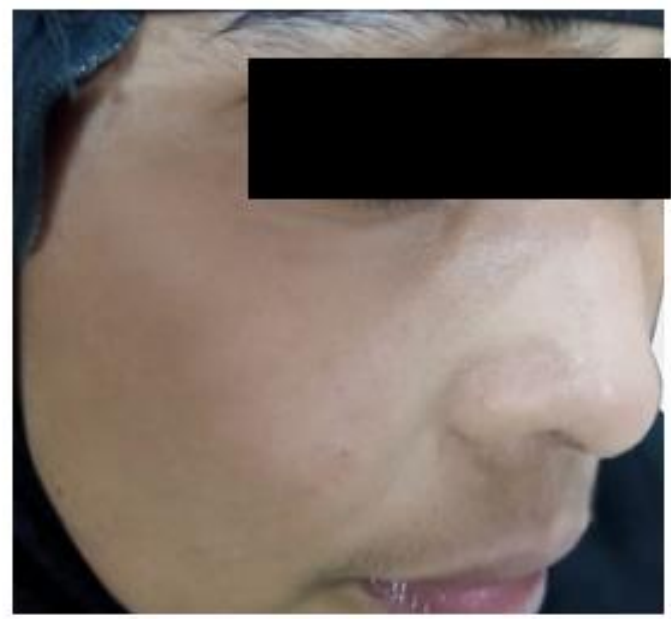

Photo (2b): Right side melasma at the end of the treatment with very good response

and week 12, improvement of pigmentation $(a, b)$ is noticeable with very good response. 


\section{DISCUSSION}

Our comparative therapeutic trial was to evaluate the efficacy of intradermal injection of tranexamic acid in melasma treatment in comparison with saline. The duration of this study was during the period from August 2019 to October 2019. The treatment protocol included three months for treatment and follow up. General examination, dermatological examination included lesion distribution, Fitzpatrick skin phototype detection. Wood's lamp examination was done for whole sixty Egyptian female patients with different types and patterns of melasma recruited from the Dermatology Outpatient Clinic of Al-Azhar University Hospitals Informed written consents were obtained from all patients.

Before and after treatment, digital photograghs were taken and mMASI score was done before, after and at the end of treatment.

In the present study, all patients were females. This was in agreement with Serra et al. (2018) and Jin et al. (2019), this disagreed with Sarkar et al. (2018), they interestingly found that $25.83 \%$ of Indian melasma patients were males. This could be due to the Indian climate and vegetables oils use after bath which may help in appearance of pigmentation in males.

The mean duration of melasma in our present study was 5 years. Blackburn et al. (2017), found persistence of melasma in $30 \%$ of cases after 10 years, and Ilknur et al. (2010) found that the mean duration of melasma was 11 years. These results disagreed with Achar and Rathi (2011) who found that the mean duration of melasma was 3.59 years. This was explained as the transient type of melasma disappears within a year of hormonal stimulus withdrawal or stopping the aggravating factors. While the long duration is due to persistent type of melasma, which maintain long time, it occurs when hyperpigmentation become deep and with continuous exposure to UVR or other unknown factors. This persistent type may be the cause of long duration of melasma in our studied patient.

Regarding the efficacy of treatment with intradermal tranexamic acid (4 $\mathrm{mg} / \mathrm{ml}$ ) on the right side of the face, there was a significant decrease in mMASI score after treatment. These results were in agreement with Sheth and Pandya (2011) and Saki et al. (2018), as they used intradermal injection of $0.05 \mathrm{ml}$ of tranexamic acid $4 \mathrm{mg} / \mathrm{ml}$ in patients with melasma weekly for 12 weeks, and they reported a significance decrease in the mMASI score after treatment. Also, Budamakuntla et al. (2013) compared the effect of microinjections application versus micro needling application of TXA in melasma patients. The scores showed better improvement in the micro needling procedure, though the difference was not statistically significant.

Moreover, Ebrahimi and Naeini (2014) studied the role of topical $3 \%$ tranexamic acid on one side of the face in patients with melasma daily for 12 weeks with a significant decrease in mMASI score. Topical TXA is temperature-stable, not UV sensitive, and does not get oxidized easily. Thus, it makes TXA a better choice when compared with hydroquinone which gets oxidized fast. 


\section{EVALUATION OF INTRADERMAL TRANXEMIC ACID INJECTION IN... ${ }^{667}$}

The intravenous administration of TXA was used by Malathi and Thappa (2013) in a dose of $500 \mathrm{mg} /$ week administered for a period of 1-2 months and $500 \mathrm{mg}$ every month as maintenance for skin lightening.

Na et al. (2013) concluded that TXA decreased melasma epidermal pigmentation and dermal changes in melasma as increasing vessel number and mast cells number. The mean lesional melanin index (MI) scores and the erythema index scores of lesional and perilesional skin significantly decreased after treatment. Skin biopsies showed significant reduction of epidermal pigmentation, vessel numbers and mast cell counts after receiving $1500 \mathrm{mg} / \mathrm{d}$ of oral TXA plus TXA topical agent twice a day for 8 weeks.

Bagherani (2015) compared the change in MASI score before and after treatment, using oral, topical and intradermally injected TXA, There was a statistically significant difference after TXA treatment, while there was no statistically significant difference among the three different treatment modalities of TXA.

The benefit role of tranexamic acid, acting as a plasmin inhibitor, prevents binding of plasminogen to the lysinebinding site by interfering with the kringle structure of the plasminogen molecule, thus inhibiting the plasminogen activator (PA) from converting plasminogen to plasmin. These were approved by molecular study of tranexamic acid effect on tyrosinase enzyme and melanogenesis in humen cultured melanocyte, and this effect appeared only in the presence of keratinocyte conditioned medium(KCM), while it had no role on tyrosinase activity in abcence of (KCM). So, TXA does not act directly on melanocytes, but inhibit melanocyte activators contained in KCM, by blocking the single-chain urokinasetype plasminogen activator (sc-uPA) and plasmin, which ultimately prevents arachidonic acid (AA) and prostaglandin (PG) synthesis (Wu et al., 2012).

TXA also suppresses angiogenesis and also inhibits neovascularization induced by basic fibroblast growth factor (bFGF) that released from plasmin. TXA also decreased mast cells as it plays an important role in melasma development.

Concerning the side effects in the present study, burning sensation and mild transient edema at the site of injection were detected as the only side effect in whole thirty patients. These agreed by Budamakuntla et al. (2013) as 50\% of the studied group had burning sensation and mild aedema with intradermal injection of tranexamic acid.

Tse and Hui (2013) revealed that the most therapies of melasma treatment act on the pre-existing melanocyte (topical agents), and eliminating pre-existing melanin pigment (peeling, IPL, laser). However, they inevitably activate melanocyte by different irritation, inflammation or by injuries to keratinocyte that lead to recurrence or postinflammatory hyperpigmentation (PIH), while tranexamic acid is the only modality that can actually prevent the activation of melanocyte by sunlight, hormonal influence, and injured keratinocyte (after UV, peeling, IPL, laser), and also prevent $\mathrm{PIH}$ at the same time. George (2016) suggested that TA has a role in PIH treatment after laser 
therapy, a common concern in people with darker skin complexions, through its antiinflammatory action. It inhibits high levels of inflammatory mediators including IL$1 \alpha$, IL-8, and PGE2 that are melanogenic and tyrosinase activity stimulators, presented in laser-treated keratinocyteconditioned medium (LT-KCM).

\section{CONCLUSION}

Intradermal tranexamic acid is safe, well tolerated and effective therapy in the treatment of melasma. Tranexamic acid solution is an available therapy, not expensive and easy to be prepared and applied. Tranexamic acid is preferred in dark skin phototypes than as it is safer and less liable to cause PIH. Tranexamic acid prevents the rapid relapse of melasma after stoppage of treatment.

\section{REFERENCES}

1. Achar A and Rathi SK (2011): Melasma: A clinico-epidemiological study of 312 cases, Indian J Dermatol., 56(4): 380-382.

2. Bagherani N (2015): The efficacy of tranexamic acid in the treatment of melasma. Dermatol Ther., 28(4): 265-265.

3. Bala HR, Lee $S$, Wong $C$, Pandya AG and Rodrigues M. (2018): Oral tranexamic acid for the treatment of melasma: a review. Dermatologic Surgery, 44(6): 814-825.

4. Blackburn S (ed) (2017): Maternal, Fetal, \& neonatal physiology-E-book: a clinical perspective. 5th edition, $\mathrm{Pb} 1$. Elsevier Health Sciences St., Louis Missori USA. pp: 153-161.

5. Budamakuntla L, Loganathan E, Suresh DH, Shanmugam S, Suryanarayan S, Dongare A, Venkataramiah LD and Prabhu N (2013): A Randomised, Open-label, Comparative Study of Tranexamic Acid Microinjections and Tranexamic Acid with Microneedling in Patients with Melasma. J Cutan Aesthet Surg., 6(3):139-43.
6. Ebrahimi B and Naeini FF (2014): Topical tranexamic acid as a promising treatment for melasma. J Res Med Sci., 19(8): 753-757.

7. Handel AC, Miot LDB and Miot HA (2014): Melasma: a clinical and epidemiological review. Anais brasileiros de dermatologia, 89(5): 771-782.

8. İlknur T, Demirtaşoğlu M, Biçak MÜ and Özkan Ş (2010): Glycolic acid peels versus amino fruit acid peels for acne. J Cosmet Laser Ther., 12(5): 242-245.

9. Jimbow K, Sarkar R, Pooja Arora P, Vijay Kumar Garg VK, Sidharth Sonthalia S, and Narendra Gokhale N (2014): Melasma update. Indian Dermatol Online J., 4: 426-435.

10. Jin $Y$, Jiang $W$, Yao $Y$, Huang $H$ and Huang J (2019): Clinical efficacy of laser combined with menstrual regulation in the treatment of female melasma: a retrospective study. Lasers Med Sci., 1-7.

11. Khurana VK, Misri RR, Agarwal S, Thole AV, Kumar $S$ and Anand T (2019): A randomized, open-label, comparative study of oral tranexamic acid and tranexamic acid microinjections in patients with melasma. Indian Journal of Dermatology, Venereology, and Leprology, 85(1): 39.

12. Malathi M and Thappa DM (2013): Systemic skin whitening/lightening agents: What is the evidence? Indian J Dermatol Venereol Leprol., 79:842-6.

13. Na JI, Choi SY, Yang SH, Choi HR, Kang HY and Park KC (2013): Effect of tranexamic acid on melasma: A clinical trial with histological evaluation. J Eur Acad Dermatol Venereol., 27:1035-9.

14. Perper M, Eber AE, Fayne R, Verne SH, Magno RJ, Cervantes J and Nouri K (2017): Tranexamic acid in the treatment of melasma: a review of the literature. American Journal of Clinical Dermatology, 18(3): 373-381.

15. Saki N, Darayesh M and Heiran A (2018): Comparing the efficacy of topical hydroquinone $2 \%$ versus intradermal tranexamic acid microinjections in treating melasma: a split-face controlled trial. J Dermatol Treatment., 29(4): 405-410. 
EVALUATION OF INTRADERMAL TRANXEMIC ACID INJECTION IN... 669

16. Sarkar R, Ailawadi $P$ and Garg $S$ (2018): Melasma in men: A review of clinical, etiological, and management issues. The Journal of Clinical and Aesthetic Dermatology, 11(2):53.

17. Sarkar R, Ailawadi P and Garg SH (2018): Melasma in Men: A Review of Clinical, Etiological, and Management Issues. J Clin Aesthet Dermatol., 11(2): 53-59.

18. Sarkar R, Gokhale N, Godse K, Ailawadi P Arya L, Sarma N, Torsekar RG, Somani VK, Arora P, Majid I, Ravichandran G, Singh M, Aurangabadkar S, Arsiwala S, Sonthalia S, Salim T and Shah S (2017): Medical Management of Melasma. A Review with Consensus Recommendations by Indian Pigmentary Expert Group. Indian Journal Dermatol., 62(6): 558-561.

19.Serra M, Bohnert K, Narda M, Granger C and Sadick N (2018): Brightening and improvement of facial skin quality in healthy female subjects with moderate hyperpigmentation or dark spots and moderate facial aging. J Drugs Dermatol: JDD, 17(12): 1310-1315.

20. Shankar K, Godse K, Aurangabadkar S, Lahiri K, Mysore V, Ganjoo A, Vedamurty M, Kohli M, Sharad J, Kadhe $G$ and Ahirrao P (2014): Evidence-based treatment for melasma: expert opinion and a review. Dermatology and Therapy, 4(2):165-86.
21. Sharma R, Mahajan VK, Mehta KS, Chauhan PS, Rawat R and Shiny TN (2017): Therapeutic efficacy and safety of oral tranexamic acid and that of tranexamic acid local infiltration with microinjections in patients with melasma: a comparative study. Clinical and Experimental Dermatology, 42(7):728-34.

22. Sheth VM and Pandya AG (2011): Melasma: a comprehensive update: part II. Journal of the American Academy of Dermatology, 65(4):699-714.

23. Sheu SL (2018): Treatment of melasma using traneximic acid: what's known and whats next. Cutis, 101(2):E7-E8.

24. Tse TW and Hui E (2013): Tranexamic acid: an important adjuvant in the treatment of melasma. J Cosmet Dermatol., 12(1): 57-66.

25. Wu S, Shi H, Wu H, Yan S, Guo J, Sun Y and Pan L (2012): Treatment of melasma with oral administration of tranexamic acid. Aesthetic Plastic Surgery, 36(4):964-70.

26.Zhang L, Tan WQ, Fang QQ, Zhao WY, Zhao QM, Gao J and Wang XW (2018): Tranexamic acid for adults with melasma: a systematic review and meta-analysis. BioMed Research International, 2018:1683414. 


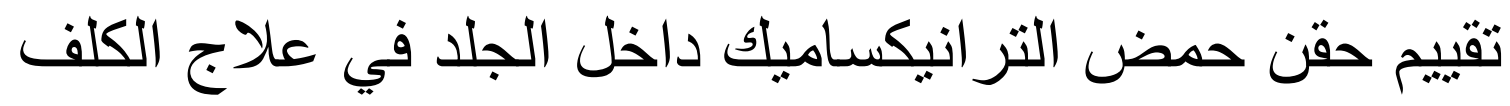 محمد فوزي سعد عويس، عبد الرؤف محمد المحسن، عماد محمود الروينى قسم الجلدية والتناسلية والذكورة، كلية الطب، جامعة الازهر، القاهرة، مصر}

E-mail: dr.fawzyewsiss@gmail.com

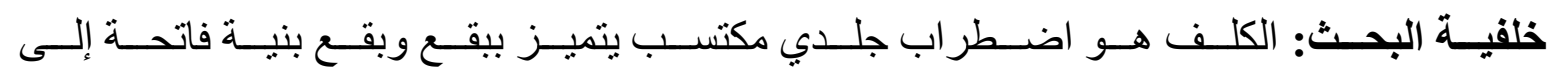

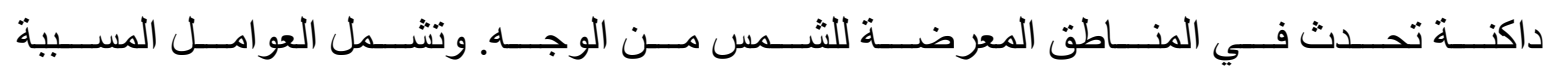

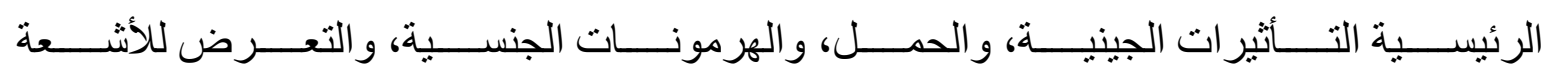

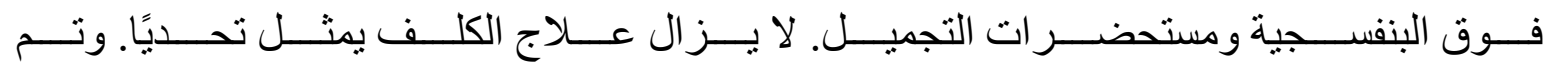

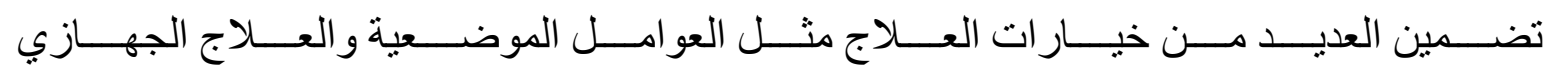

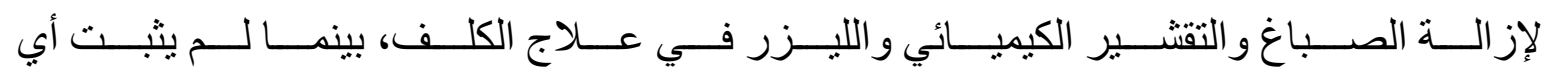

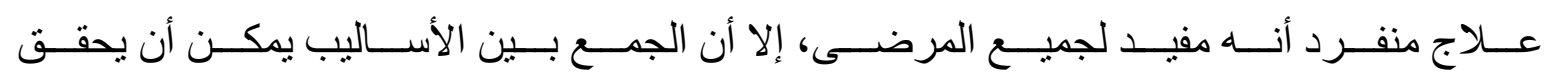
مزيدًا من التحكم خاصة في الحالات الصعبة.

الهـــف مـن البحــث: تقيــيم فعاليــة حقـن حمـض التر انيكســاميك داخـل الأدمـة فـي عـلاج الكلف.

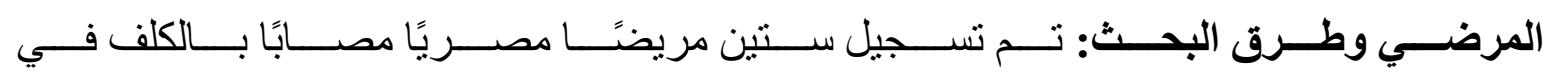

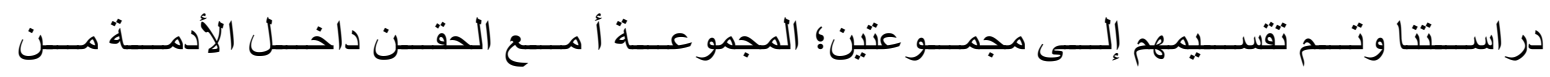

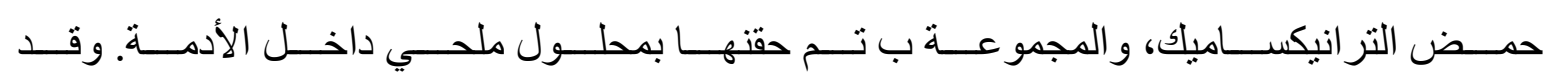

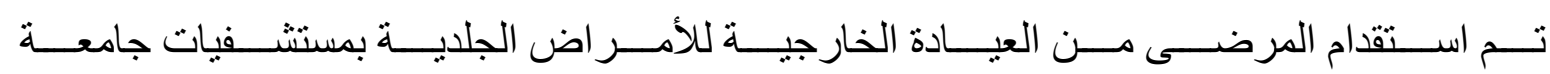
الأزهر في الفترة من أغسطس 2019 إلى أبريل 2020.

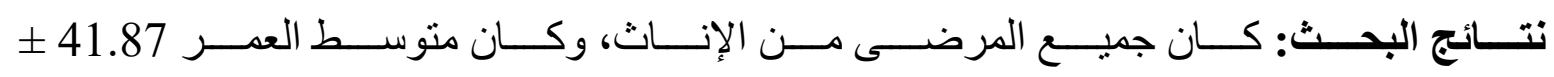

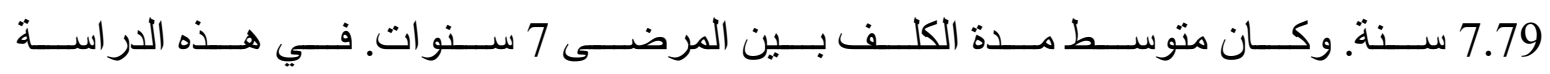

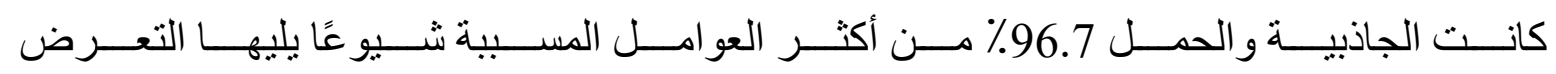

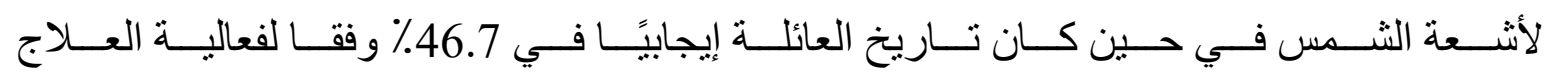

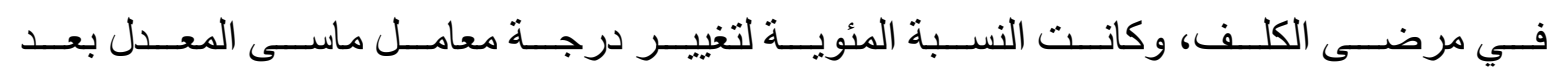

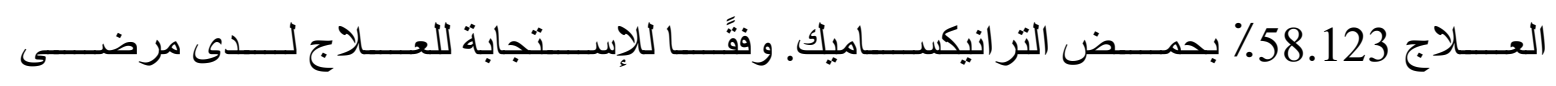




\section{EVALUATION OF INTRADERMAL TRANXEMIC ACID INJECTION IN... ${ }^{671}$}

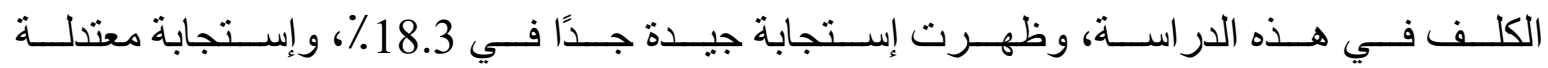

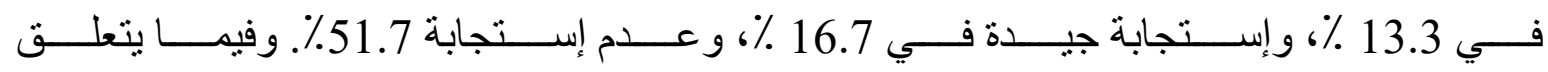

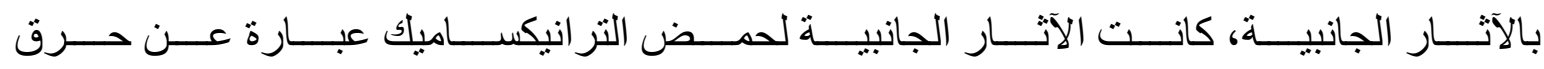

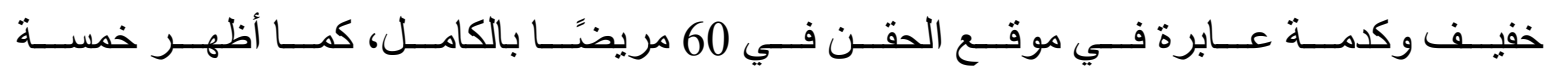
مرضى فرط تصبغ ما بعد الالتهاب (8.3 \%).

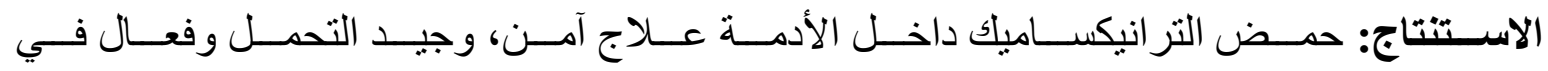

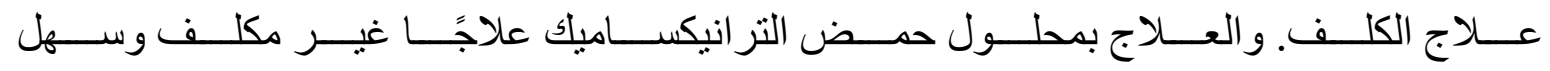

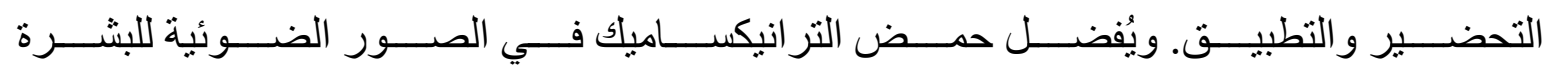

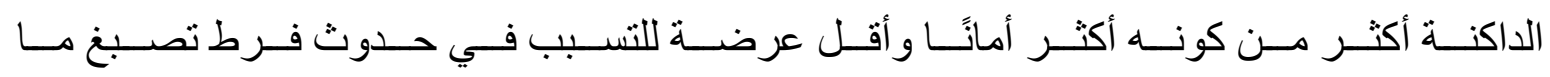

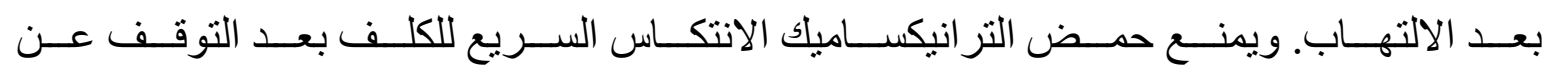
العلاج. الكلمات الالة : حمض التر انيكساميك - الكلف . 